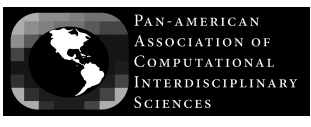

Journal of Computational Interdisciplinary Sciences (2009) 1(2): 73-82

(C) 2009 Pan-American Association of Computational Interdisciplinary Sciences

ISSN 1983-8409

http://epacis.org

\title{
Riddled basins in complex physical and biological systems
}

\author{
Ricardo Luiz Viana ${ }^{1}$ Sabrina Camargo², Rodrigo F. Pereira1, Marcos César Vergés ${ }^{1}$, \\ Sérgio Roberto Lopes ${ }^{1}$ and Sandro Ely S. Pinto ${ }^{3}$ \\ Manuscript received on November 12, 2008 / accepted on January 10, 2009
}

\begin{abstract}
Complex systems have typically more than one attractor, either periodic or chaotic, and their basin structure ultimately determines the final-state predictability. When certain symmetries exist in the phase space, their basins of attraction may be riddled, which means that they are so densely intertwined that it may be virtually impossible to determine the final state, given a finite uncertainty in the determination of the initial conditions. Riddling occurs in a variety of complex systems of physical and biological interest. We review the mathematical conditions for riddling to occur, and present two illustrative examples of this phenomenon: coupled Lorenz-like piecewise-linear maps and a deterministic model for competitive indeterminacy in populations of flour beetles.
\end{abstract}

Keywords: riddled basins, basins of attraction, complex systems, chaotic attractors.

Correspondence to: Ricardo Luiz Viana

1 Departamento de Física, Universidade Federal do Paraná, 81531-990 Curitiba, PR, Brazil.

2 Instituto de Física, Universidade de São Paulo, 05315-970 São Paulo, SP, Brazil.

3 Departamento de Física, Universidade Estadual de Ponta Grossa, 84032-900 Ponta Grossa, PR, Brazil.

E-mails: viana@fisica.ufpr.br / sabrina@fisica.ufpr.br / pereira@fisica.ufpr.br / mcverges@fisica.ufpr.br / lopes@fisica.ufpr.br / desouzapinto@gmail.com 


\section{INTRODUCTION}

Chaotic dynamical systems having certain symmetries and quite general mathematical properties may present basins of attraction densely intertwined, a phenomenon called riddling (for a recent review covering theoretical and experimental aspects of riddling see Ref. [1]). In this case the system has a chaotic attractor A whose basin of attraction is riddled with "holes" (in a measuretheoretical sense) belonging to the basin of another (non necessarily chaotic) attractor B [2]. In other words, riddling means that every point in the basin of attractor $A$ has pieces of the basin of attractor $\mathrm{B}$ arbitrarily nearby. The basins are called intermingled, when each basin is riddled with holes belonging to the other basin. For intermingled basins there must be at least two attractors lying in different invariant subspaces [2, 3].

Basin riddling affects our ability of predicting what attractor the trajectory originating from a given initial condition asymptotes to. Let $\mathrm{P}$ be an arbitrary point belonging to the basin of the chaotic attractor $A$. If the basin of $A$ is riddled by the basin of the other attractor $\mathrm{B}$, then a small ball of radius $\epsilon$ centered at $P$ has a nonzero fraction of its volume belonging to the basin of $\mathrm{B}$, irrespective of how small the radius $\epsilon$ may be. Hence, if we regard this $\epsilon$-ball as an uncertainty neighborhood related to the (numerical or experimental) determination of the initial condition, the resulting trajectory has always a positive probability of falling into the basin of the other attractor. In other words, the probability of escaping from the basin of attractor $A$ is nonzero for every uncertainty $\epsilon$. Consequently, in the presence of riddling, the task of predicting what will be the final state of the system becomes much more difficult than in the cases of fractal basins, where the boundary is a fractal curve and the uncertain fraction scales with $\epsilon$ as a power-law [4].

There are many examples of riddling in dynamical systems of physical and biological interest, as a forced double-well Duffing oscillator $[5,6,7]$, coupled nonlinear electronic circuits $[8,9]$, coupled elastic arches [10], ecological population models [11], learning dynamical systems [12], chemical reactions of the Belouzov-Zhabotinsky type [13], and in models of interdependent open economies [14]. In this paper we describe applications of riddled and intermingled basins in physical and biological complex systems.

This paper is organized as follows: in Section 2 we review the mathematical conditions for the existence of riddled basins. Section 3 deals with riddled basins in coupled piecewise-linear maps which can be viewed as a low-dimensional reduction of coupled Lorenz equations, for which riddling is thought to occur.
Section 4 brings an application of riddling in a dynamical system of biological interest, describing the competitive indeterminacy for two species of a flour beetle, and which presents intermingled basins. The last Section contains our conclusions.

\section{MATHEMATICAL CONDITIONS FOR THE EXISTENCE OF RIDDLED BASINS}

Let $\mathrm{H}$ be the phase space in which a discrete-time map $x_{n+1}=$ $F\left(x_{n}\right)$ is defined. Continuous-time flows can be also described by $F$, if Poincaré sections are taken. A closed subset $\mathrm{A} \in \mathrm{H}$ is said to be an attractor of $F$ if it satisfies the following conditions:

(i) A has a basin of attraction, denoted $\beta(\mathrm{A})$, of positive Lebesgue measure (volume) in the phase space $\mathrm{H}$;

(ii) $\mathrm{A}$ is a compact set with a dense orbit. In the Milnor definition of attractor, the basin of attraction does not need to include the whole neighborhood of the attractor.

If the basin of attraction of $\mathrm{A}$ has positive Lebesgue measure, we call A a weak Milnor attractor [15].

The basin of a chaotic attractor $\mathrm{A}$ is riddled if its complement intersects every disk(in the sense of phase-space volumes of all sizes) of the phase space $\mathrm{H}$ in a set of positive Lebesgue measure [2]. When the basin of attraction of $A$ is riddled with holes belonging to the basin of another attractor $\mathrm{B}$, we can say that, if a randomly chosen point has a positive probability of being in $\beta(\mathrm{A})$, then it also has positive probability of not being in $\beta(\mathrm{A})$. In the latter case, the point belongs to the other basin of attraction $\beta(\mathrm{B})$.

This measure-theoretical definition implies the following set of conditions under which riddled basins occur in a dynamical system [6]:

1. there is an invariant subspace $\mathrm{M} \in \mathrm{H}$;

2. the dynamics on $M$ has a chaotic attractor $A$

3. there is another attractor $\mathrm{B}$ not belonging to $\mathrm{M}$;

4. the attractor $\mathrm{A}$ is transversely stable in $\mathrm{H}$, i.e. for typical orbits on the attractor the Lyapunov exponents for infinitesimal perturbations along the directions transversal to the invariant subspace $\mathbf{M}$ are all negative;

5. a set of unstable periodic orbits embedded in A is transversely unstable. As a consequence, at least one of the Lyapunov exponents along directions transverse to $\mathrm{M}$ experiences positive finite-time fluctuations. 
Condition $\mathbf{1}$ is a consequence of the system having some symmetry which enables it to display an invariant subspace $\mathbf{M}$, in the sense that, once an initial condition is exactly placed on $\mathbf{M}$, the resulting trajectory cannot escape from $\mathbf{M}$ for further times. To have riddling, it is necessary to have a dense set of points with zero Lebesgue measure in the attractor lying in the invariant subspace which are transversely unstable, thus it is necessary that this attractor be chaotic (condition 2). The existence of another attractor (condition $\mathbf{3}$ ) is necessary for the basin of an attractor to be riddled with holes belonging to the basin of this second attractor.

If the transverse Lyapunov exponents of typical orbits lying in the invariant manifold $M$ are all negative (condition $\mathbf{4}$ ), then $A$ is an attractor at least in the weak Milnor sense, and its basin has positive Lebesgue measure. This can be verified by computing the maximal Lyapunov exponent along a transversal direction to $\mathrm{M}$ :

$$
\lambda_{\perp}=\lim _{n \rightarrow \infty} \tilde{\lambda}_{\perp}\left(x_{0}, n\right)<0,
$$

where

$$
\tilde{\lambda}_{\perp}\left(x_{0} ; n\right)=\frac{1}{n} \ln \left\|D f^{n}\left(x_{0}\right) \cdot v_{\perp}\right\|,
$$

is the maximal time- $n$ Lyapunov exponent along a transversal direction (specified by the singular vector $v_{\perp}$ ) with respect to the invariant manifold $\mathrm{M}, D F^{n}\left(x_{0}\right)$ being the Jacobian matrix of the $n$ times iterated map $F$, with entries evaluated at an initial condition $x_{0} \in \mathrm{A}$.

Condition $\mathbf{5}$ states that, while the invariant manifold $\mathbf{M}$ is still transversely stable, there will be trajectories on the attractor $\mathrm{A}$ that are transversely unstable. Verifying condition $\mathbf{5}$, on the other hand, would require the determination of a transversely unstable periodic orbit embedded in the attractor $\mathrm{A}$. A consequence of these transversely unstable orbits is that there will be typically a number of positive values of the finite-time transversal Lyapunov exponent, or $\tilde{\lambda}_{\perp}\left(x_{0}, n\right)>0$. Hence, this condition can be statistically verified by considering the probability distribution of finite-time transversal exponents $P\left(\tilde{\lambda}_{\perp}\left(x_{0}, n\right)\right)$.

The existence of positive values of $\tilde{\lambda}_{\perp}\left(x_{0}, n\right)$ - condition $\mathbf{5}$ for riddling - implies that there is a fraction of positive values of $\tilde{\lambda}_{\perp}\left(x_{0}, n\right)$ for initial conditions $x_{0}$ randomly chosen in the attractor $\mathrm{A}$, i.e.

$$
f(n)=\int_{0}^{\infty} P\left(\tilde{\lambda}_{\perp}(n)\right) d \tilde{\lambda}_{\perp}(n)>0 .
$$

assuming that the probability is properly normalized.

\section{RIDDLED BASINS IN LORENZ-LIKE MAPS}

The modern age of nonlinear dynamics begun in 1963, when Ed Lorenz investigated the dynamics generated by a three-mode reduction of the equations governing thermal convection, that is one of the mechanisms underlying climate changes [16]. Lorenz realized that the chaotic dynamics exhibited by the three-mode differential equations

$$
\begin{aligned}
& \frac{d x}{d t}=10(y-x), \\
& \frac{d y}{d t}=x(28-z)-y, \\
& \frac{d z}{d t}=x y-\frac{8}{3} z,
\end{aligned}
$$

could be understood in terms of a simpler, low-dimensional discrete map. The so-called Lorenz map is obtained by sampling the local maxima of one of the evolving variables, $h_{n}=$ $\max _{t=t_{n}}\{z(t)\}$, so as to yield a first return map $h_{n+1}=L\left(h_{n}\right)$, which is unimodal with a cusp [Fig. 1(a)]. Such maps display a similar dynamical behavior as the tent map $\left(x_{n+1}=\right.$ $\left.1-2\left|x_{n}-1 / 2\right|\right)$ and, in fact, this similarity was used by Lorenz to explain the erratic behavior of orbits belonging to the famous "butterfly attractor" in the three-dimensional phase space [16].

Another piecewise-linear approximation to the Lorenz map is provided by the so-called bungalow-tent map, which has out of four linear segments instead of two, as in the tent function [17, 18]:

$$
\begin{gathered}
x_{n+1}=f_{a}\left(x_{n}\right)= \\
= \begin{cases}\frac{1-a}{a} x_{n}, & \text { if } x_{n} \in[0, a), \\
\frac{2 a}{1-2 a} x_{n}+\frac{1-3 a}{1-2 a}, & \text { if } x_{n} \in\left[a, \frac{1}{2}\right), \\
\frac{2 a}{1-2 a}\left(1-x_{n}\right)+\frac{1-3 a}{1-2 a}, & \text { if } x_{n} \in\left[\frac{1}{2}, 1-a\right), \\
\frac{1-a}{a}\left(1-x_{n}\right), & \text { if } x_{n} \in[1-a, 1],\end{cases}
\end{gathered}
$$

where $a \in\left(0, \frac{1}{2}\right)$ is a control parameter. For $a=\frac{1}{3}$ we obtain the tent map. A linear coordinate transformation $h=22 x+32$ and the choice $a=0.45$ furnish a better approximation of the Lorenz map in comparison with the tent function [Fig. 1(b)]. In the following we will refer to the former as the piecewise-linear Lorenz map.

The two fixed points of the bungalow map are $P_{1}: x_{1}^{*}=0$ and the right corner point $P_{2}: x_{2}^{*}=1-a$. For all values of the control parameter $a$, the fixed points are unstable 


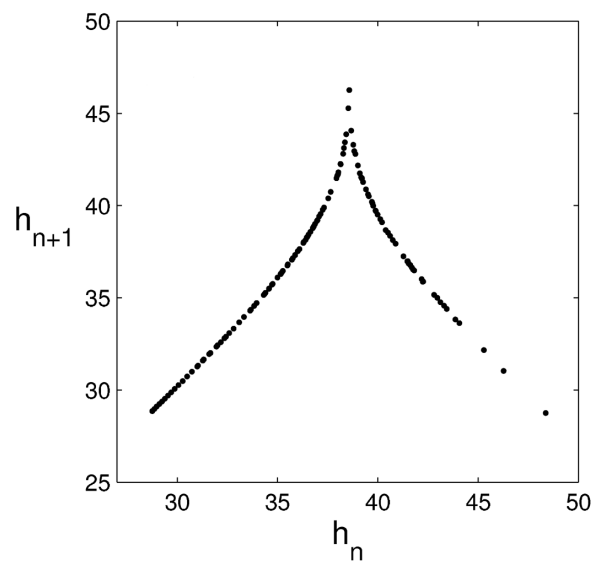

(a)



(b)

Figure 1 - (a) Lorenz map. (b) Bungalow-tent map for $a=0.45$.

because the eigenvalues $\delta_{1,2}=(a-1) / a$ are greater than 1 in modulus. Although the map is non-smooth at the fixed point $x_{2}^{*}$, we can define the map derivative at the right-hand site of $x_{2}^{*}$, because this point belongs to the interval $[1-a .1]$. Thanks to the piecewise-linearity of this map, it is possible to obtain analytically its Lyapunov exponent for any value of $a$, namely $[17,18]$ :

$$
\lambda(a)=\frac{1-a}{2-3 a} \ln \left(\frac{1-a}{a}\right)+\frac{1-2 a}{2-3 a} \ln \left(\frac{2 a}{1-2 a}\right) .
$$

In particular, for $a=1 / 3$ (tent map) we obtain $\lambda=\ln 2$, and, for other values of $a$, the Lyapunov exponent is always positive, displaying a smooth dependence with $a$. For the approximation to the Lorenz map which results from $a=0.45$, we have $\lambda=0.5078$.

Systems of coupled Lorenz equations have been found numerically to exhibit a suggestive evidence for riddled basins [19], although without a mathematical proof. Such an investigation is indeed quite difficult in view of the high dimensionality of the system and of the rather strong mathematical requirements necessary to characterize riddled basins, as we have seen in the previous Section. Hence we can resort to the one-dimensional reduction performed by taking the Lorenz map and its piecewise-linear counterpart. Hence we can study the presence of riddled basins in coupled Lorenz equations starting from its simplest possible version, which is the coupling of piecewise-linear Lorenz maps in the form

$$
\begin{aligned}
& x_{n+1}=f_{a}\left(x_{n}\right)+\delta\left(x_{n}-y_{n}\right), \\
& y_{n+1}=f_{a}\left(y_{n}\right)+\epsilon\left(y_{n}-x_{n}\right),
\end{aligned}
$$

where $f_{a}$ is given by Eq. (7), and $\delta$ and $\varepsilon$ are coupling strengths which can be different when the coupling is asymmetric, and even vanish for unidirectional coupling (a master-slave configuration). As we will see, the dynamics of the system depends essentially on their sum $d \equiv \delta+\varepsilon$.

It has been known for a long time that two identical coupled chaotic systems, in spite of their characteristic sensitivity on the initial condition, can attain a completely synchronized state characterized by $x_{n}=y_{n}$ for all times $n[20,21]$. In terms of the two-dimensional phase space $\mathrm{H}$ of the coupled system, the synchronized state defines a synchronization subspace $\mathbf{M}$, which is the straight line $x=y$. The orbit in the synchronized state is obviously the same as a chaotic orbit from the uncoupled maps. Hence, it turns out that $\mathbf{M}$ is an invariant subspace, thus fulfilling condition $\mathbf{1}$ for riddling. Moreover, there is a chaotic attractor A embedded in the synchronization subspace (condition 2), such that the fixed points in $\mathrm{M}$ are $P_{1}=0$ and $P_{2}=1-a$, belonging to the intervals $[0, a)$ and $[1-a, 1]$, respectively. In this case, there is only one transversal direction to $\mathrm{M}$. Besides the synchronized state, there exists another attractor $\mathrm{B}$ off the manifold $\mathrm{M}$, that is the attractor at infinity (condition $\mathbf{3}$ ).

In order to verify condition $\mathbf{4}$ for riddling, we have to investigate for which values of the coupling coefficient $d$ the transversal Lyapunov exponent is negative. The exponent along the transversal direction, on the other hand, can be obtained by applying Birkhoff ergodic theorem as [15]:

$$
\begin{aligned}
\lambda_{\perp}= & \frac{1}{2-3 a}\left[a \ln \left|\frac{1-a}{a}-d\right|\right. \\
& +\frac{1-2 a}{2} \ln \left|\frac{2 a}{1-2 a}-d\right|
\end{aligned}
$$




$$
\begin{aligned}
& \left.+\frac{1-2 a}{2} \ln \left|\frac{-2 a}{1-2 a}-d\right|\right] \\
& +\frac{1-2 a}{2-3 a} \ln \left|\frac{-(1-a)}{a}-d\right|,
\end{aligned}
$$

where we have used the invariant density of the isolated map $[17,18]$.

In Figure 2 we plot the transversal Lyapunov exponent as a function of the coupling strength sum $d=\delta+\varepsilon$ for $a=0.45$. There are out of four intervals of $d$ for which the transversal Lyapunov exponent is negative, thus fulfilling Condition 4 for riddling. The boundaries of these intervals are the given by the values of $d$ for which $\lambda_{\perp}=0$, yielding transcendental equations in view of (11). The intervals characterized by negative transversal exponents for the piecewise-linear Lorenz case $(a=0.45)$ are $I_{1}=(-1.224,-1.220)$ and $I_{2}=(0.689,1.707)$.

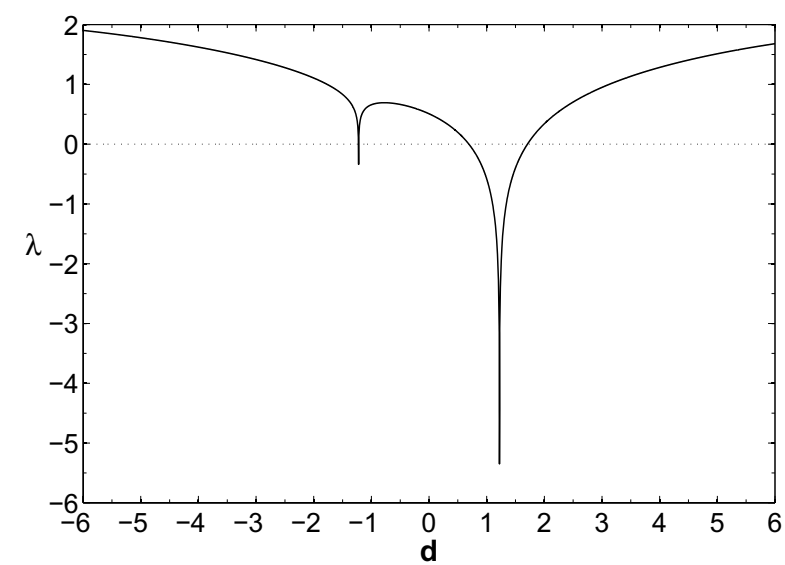

Figure 2 - Transversal Lyapunov exponent for the synchronized attractor of coupled piecewise-linear Lorenz maps with $a=0.45$ (as a function of the coupling strength $d$.

We can now verify condition $\mathbf{5}$ for riddling, that demands that there exist transversely unstable fixed points in the synchronized attractor $\mathbf{A}$. In principle, any periodic orbit embedded in A can be transversely unstable, so we start by the fixed points. Thanks to the piecewise-linearity of the bungalow-tent map, we can compute analytically the eigenvalues of the fixed points along transversal directions to the synchronization subspace. In Figure 3 we plot the moduli of the transversal eigenvalue $\mu_{2}$ as a function of the coupling parameter $d$ for the fixed points $P_{1}$ and $P_{2}$ of the coupled maps. Regardless of the value taken on by $d$, there will be always some transversely unstable fixed point, i.e. there is at least one transversal eigenvalue having modulus greater than the unity.
For the fixed point $P_{1}=0$ this interval is $d_{1}<d<d_{2}$, where

$$
d_{1,2}=\left(\frac{1-a}{a}\right) \mp 1,
$$

and for $P_{2}=1-a,-d_{2}<d<-d_{1}$. For $a=0.45$, as in Figure 3 , these intervals are $I_{3}=(-2.23,-0.22)$ and $I_{4}=(0.22,2.23)$ for the fixed points $P_{1}$ and $P_{2}$, respectively.

Summing up, since condition $\mathbf{5}$ is verified for any $d$, the only relevant condition for riddling is $\mathbf{4}$, i.e. the coupling strength sum $d$ must take on a value belonging to the intervals $I_{1}$ or $I_{2}$ such that we have riddled basins of attraction.

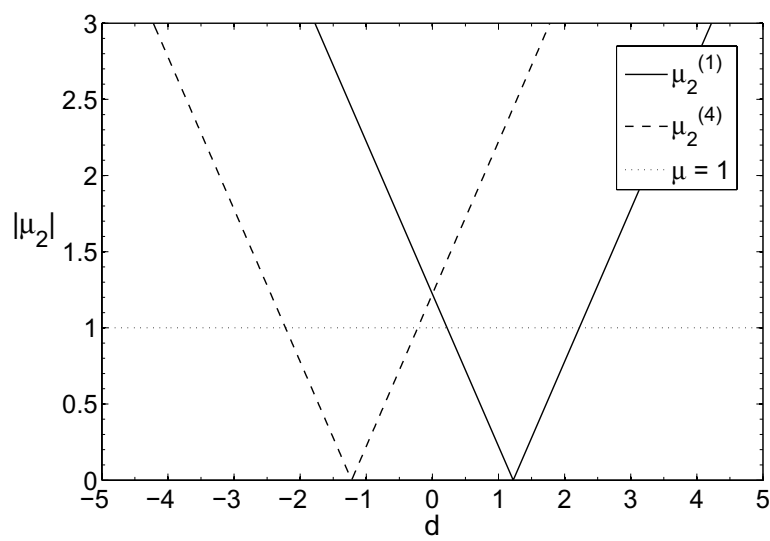

Figure 3 - Moduli of the transversal eigenvalue of the coupled piecewise-linear Lorenz maps as a function of the coupling strength $d$ for the fixed points $P_{1}=0$ and $P_{2}=1-a$.

An example of a non-riddled basins of synchronization is provided by Figure 4(a), where the black pixels represent initial conditions in the phase plane which asymptote to the synchronized state $x_{n}=y_{n}$, whereas white pixels represent initial conditions generating orbits which go to infinity. In fact, the black and white regions do not seem to be densely intertwined, as required for riddled basins, and are actually fractal ones, with a nonetheless involved basin boundary structure. A riddled basin is exemplified in Figure $4(\mathrm{~b})$, for which the value $(d=1.5)$ of the coupling strength is such that $\lambda_{T}<0$ (condition 4 ). The black and white regions are densely intermixed, such that for any point belonging to the basin of the synchronized attractor there exists a neighborhood containing points belonging to the basin of infinity.

\section{INTERMINGLED BASINS IN A COMPETITION TWO-SPECIES SYSTEM}

Experiments on the competition of two species of flour beetles, Tribolium castaneum and Tribolium confusum resulted in the eventual extinction of either one of the two competitors [22]. However, 


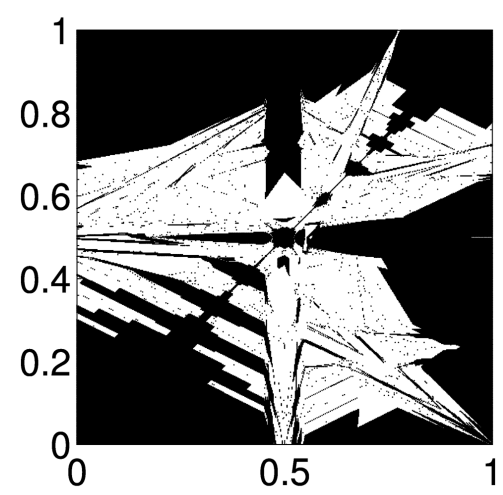

(a)

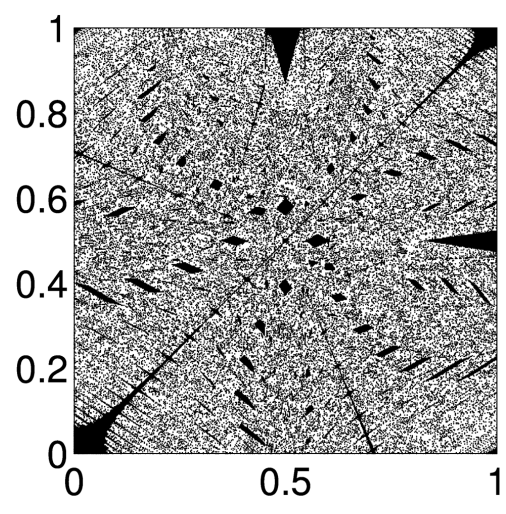

(b)

Figure 4 - Initial conditions in the phase plane for $a=0.45$ and (a) $d=0.5$ and (b) $d=1.5$. The black pixels represent initial conditions which converge to a synchronized state, whereas white pixels are initial conditions generating orbits going to infinity.

the particular species to become extinct was found to be extremely sensitive to the initial population and the environmental conditions (such as temperature and humidity) prevailing during the realization of the experiment [23]. Moreover, there are also experimental evidences of chaotic behavior in the time evolution of the populations of single species of Tribolium [24]. Hence we may regard the extinction of either species as an asymptotic state with a well-defined attractor in the phase space [25]. The observed extreme sensitivity on the initial condition has led to the hypothesis that the basins of these attractors are riddled [26].

In fact, the basins of attraction are intermingled, for each basin is riddled with holes belonging to the other basin. In this case the two coexisting attractors must lie in different invariant subspaces, and the basin of each attractor is pierced with holes containing initial conditions belonging to the basin of the other attractor [2, 3]. Moreover, these basins are so intertwined that, given an initial condition with a finite uncertainty, the final state cannot be predicted. The sensitivity observed in the Tribolium $s p$. experiments suggests that a mathematical model describing the problem should exhibit intermingled basins, since the initial population in the experiments correspond to an initial condition which is unavoidably plagued with some uncertainty, and thus the outcome becomes uncertain, even if the accuracy is very large in determining the initial condition [25, 26].

Hofbauer and coworkers [25] have developed a class of twodimensional models in which we consider two species with populations $x_{1}(n)$ and $x_{2}(n)$ at (discrete) times $n=0,1,2, \ldots$, which labels the insect generation, and satisfying identical evolution equations. We can adapt our conditions for riddling to the case of intermingled basins in this two-dimensional phase space $\mathrm{H}$, as follows: 1a. there are two invariant one-dimensional subspaces $M_{0}$ and $\mathrm{M}_{1}$ in the phase plane;

2a. the dynamics on the invariant subspaces $\mathrm{M}_{0}$ and $\mathrm{M}_{1}$ have chaotic attractors $A$ and $B$, respectively;

3a. the attractors $A$ and $B$ are transversely stable in the phase plane, i.e. for typical orbits on the attractors the Lyapunov exponent for infinitesimal perturbations along the direction transversal to the invariant subspaces $\mathrm{M}_{0}$ and $\mathrm{M}_{1}$, respectively, is negative;

4a. a set of unstable periodic orbits embedded in the chaotic attractors $\mathrm{A}$ and $\mathrm{B}$ are transversely unstable. As a consequence, along the direction transversal to $\mathrm{M}_{0}$ and $\mathrm{M}_{1}$, the Lyapunov exponent experiences positive finite-time fluctuations.

The Hofbauer model starts from the map equations

$$
\begin{aligned}
& x_{1}(n+1)=x_{1}(n) \Phi\left(x_{1}(n)+x_{2}(n)\right), \\
& x_{2}(n+1)=x_{2}(n) \Phi\left(x_{1}(n)+x_{2}(n)\right),
\end{aligned}
$$

where $\Phi($.$) is a function compatible with three biological requi-$ rements: (i) the proportion of each species does not change with time, i.e. $x_{2}(n+1) / x_{1}(n+1)=x_{2}(n) / x_{1}(n)$; (ii) the total population $x=x_{1}+x_{2}$ in a generation depends only on the value at its previous generation; and (iii) $\partial \Phi / \partial x_{1}<0$ and $\partial \Phi / \partial x_{2}<0$.

Perturbations of Eqs. (13)-(14) are included in the model so as to bring about competition effects that may lead to species extinction:

$$
\begin{aligned}
x_{1}(n+1)= & x_{1}(n) \Phi\left(x_{1}(n)+x_{2}(n)\right) \\
& {\left[1+\kappa x_{1}(n) G\left(x_{1}(n), x_{2}(n)\right)\right], }
\end{aligned}
$$




$$
\begin{aligned}
x_{2}(n+1)= & x_{2}(n) \Phi\left(x_{1}(n)+x_{2}(n)\right) \\
& {\left[1-\kappa x_{2}(n) G\left(x_{1}(n), x_{2}(n)\right)\right], }
\end{aligned}
$$

where $0<\kappa<1$ stands for the strength of the competition between species and, for simplicity, we assume that $G($.) depends only on the total population $x=x_{1}+x_{2}$. Changing variables from $\left(x_{1}, x_{2}\right)$ to $\left(x, y \equiv x_{1} / x\right)$ we obtain, from Eqs. (15)-(16), the following two-dimensional map

$$
\begin{aligned}
& x(n+1)=T(x(n)) \equiv x(n) \Phi(x(n)), \\
& y(n+1)=y(n)+\kappa y(n)(1-y(n)) g(x(n)),
\end{aligned}
$$

where $g(x)=x G(x y, x(1-y))$ satisfies the mathematical requirements stated in Ref. [25]. Within the class of twodimensional discrete models defined by Eqs. (17)-(18) both functions $T$ (or $\Phi$ ) and $g$ (or $G$ ) have to be determined taking into account the possibility of riddled basins. There must be two possible outcomes: $y=0$ (extinction of the $x_{1}$ species) or $y=1$ (extinction of the $x_{2}$ species). These are the only attractors of the two-dimensional map in the phase plane, denoted as $\mathrm{A}$ and $\mathrm{B}$, respectively. Moreover, their basins of attractions $\beta(\mathrm{A})$ and $\beta$ (B) must be riddled (in fact intermingled), what poses additional requirements in the formulation of the model.

The evolution of the total population, governed by the onedimensional map $T(x)$, must be chaotic in order to fulfill condition 2a for intermingled basins, since the only way to have an infinite number of unstable periodic orbits within an attrac-

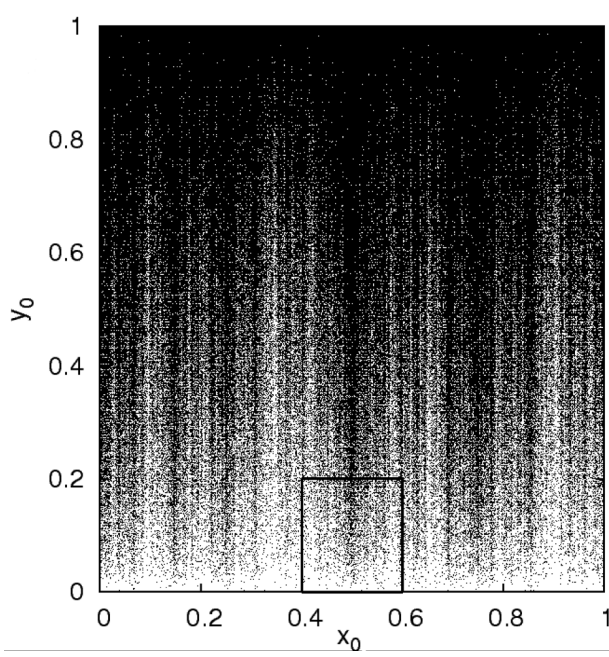

(a) tor is to ensure the existence of a dense chaotic orbit lying in the invariant manifold. The transversal dynamics (18) has two invariant subspaces: $\mathrm{M}_{0}=\{(x, y=0) \mid x \in[0,1]\}$ and $\mathrm{M}_{1}=\{(x, y=1) \mid x \in[0,1]\}$, what fulfills condition $\mathbf{1 a}$. The specific function $g(x)$, on the other hand, must be chosen so as to warrant the proper transverse stability conditions

\section{$\mathbf{3 a}$ and $\mathbf{4 a}$}

The case for which $T(x)=3 x(\bmod 1)$ and $g(x)=$ $\cos (2 \pi x)$ was previously studied by Kan [26], who proved the existence of intermingled basins when $\kappa=1 / 32$. This proof has been extended to the case $0<\kappa<1$ by Hofbauer and collaborators [25]. In the present paper we consider a slightly modified version of Kan's model, by choosing

$x(n+1)=4 x(n)(1-x(n))$,

$y(n+1)=y(n)+\kappa y(n)(1-y(n)) \cos (3 \pi x(n))$.

such that the dynamics in each invariant subspace is strongly chaotic (transitive).

A representative example of the basins of attraction exhibited by this system is depicted in Figure 5(a) for $\kappa=0.3$. A numerical approximation of the basin of the attractor at $M_{0}\left(M_{1}\right)$ is represented by the white (black) pixels, and show a fine structure, with tongues of a basin approaching the other attractor at arbitrarily small distances. Moreover, the tongue-like structure of each basin is self-similar, as suggested by the magnification shown in Figure 5(b).

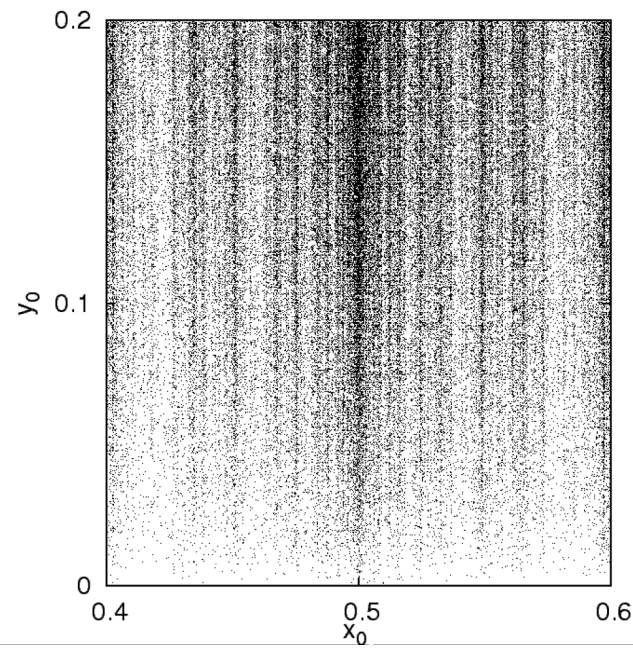

(b)

Figure 5 - Basins of the synchronized attractor (white pixels) and of the attractor at infinity (black pixels) when $\kappa=0.3$. (b) Magnification of a small region of (a). 
In order to discuss conditions $\mathbf{3 a}$ and $\mathbf{4 a}$ for intermingled basins in a quantitative setting, we deal with the finite-time Lyapunov exponents of the two-dimensional map defined by Eqs. (19)(20). If either attractor has a riddled basin, it must be transversely stable (condition $\mathbf{3 a}$ ), such that it is necessary that the infinitetime transversal Lyapunov exponent be negative for $\mathrm{A}$ and $\mathrm{B}$ :

$$
\begin{aligned}
\lambda_{\perp} & =\lim _{n \rightarrow \infty} \tilde{\lambda}_{\perp}(x(0), y(0), n) \\
& =\lim _{n \rightarrow \infty} \frac{1}{n} \sum_{i=1}^{n} \ln \left\|D F\left(x_{i}, y_{i}\right) \cdot e_{y}\right\|<0,
\end{aligned}
$$

where $D F$ is the map Jacobian. In addition, it is also required that both $\mathrm{A}$ and $\mathrm{B}$ must contain transversely unstable orbits (condition 4a). This implies the existence of positive and negative fluctuations of the finite-time transversal exponent, $\tilde{\lambda}_{\perp}(x(0), y(0), n)$, what makes useful to work with the probability distribution $P\left(\tilde{\lambda}_{\perp}(x(0), y(0), n)\right)$.

The probability distribution function (PDF) we have numerically obtained for typical chaotic orbits in both attractors is fitted by a Gaussian distribution, with small (and statistically not significant) deviations at its tails. For a Gaussian distribution, it turns out that the infinite-time Lyapunov exponent along the transversal direction is the average finite-time exponent:

$$
\begin{gathered}
\left\langle\tilde{\lambda}_{\perp}(x(0), y(0), n)\right\rangle \\
=\int_{-\infty}^{+\infty}, \tilde{\lambda}_{\perp}(n) P\left(\tilde{\lambda}_{\perp}(x(0), y(0), n)\right) d \tilde{\lambda}_{\perp}(n)=\lambda_{\perp},
\end{gathered}
$$

provided the PDF is normalized.

In Figure 6(a) we depict (in gray-scale) the dependence on the parameter $\kappa$ of the numerically obtained probability distribution function $P\left(\tilde{\lambda}_{\perp}\right)(x(0), y(0), n)$ for the time-24 transverse Lyapunov exponents. The average of these PDFs are always negative for any $\kappa$, hence $\tilde{\lambda}_{\perp}<0$ (condition $\mathbf{3 a}$ ). The widths of the PDFs increase with $\kappa$, with a marked asymmetry toward negative values of the exponent, as shown by the computed moments of the PDFs as a function of $\kappa$ [Fig. 6(b)]. We have used in these computations, both typical chaotic orbits in the attractor $\mathrm{A}$ and atypical unstable period- $p$ orbits embedded in A [27], with similar results for the attractor $\mathrm{B}$.

Verifying the condition $\mathbf{4 a}$ for intermingled basins amounts to observe a positive fraction of positive values of $\tilde{\lambda}_{\perp}(n)$ for initial conditions $(x(0), y(0))$ randomly chosen in the attractor $\mathrm{A}$ or B [cf. Eq. (3)]. If the PDFs are such that half of their values are positive sign, there results that $f(n)=1 / 2$, and the infinite-time transversal exponent vanishes $\left(\lambda_{\perp}=0\right)$, the attractor losing transversal stability (a blowout bifurcation). We remark that the occurrence of a blowout bifurcation marks the endpoint of riddling, since after that the invariant chaotic sets $\mathrm{A}$ or $\mathrm{B}$ become transversely unstable.

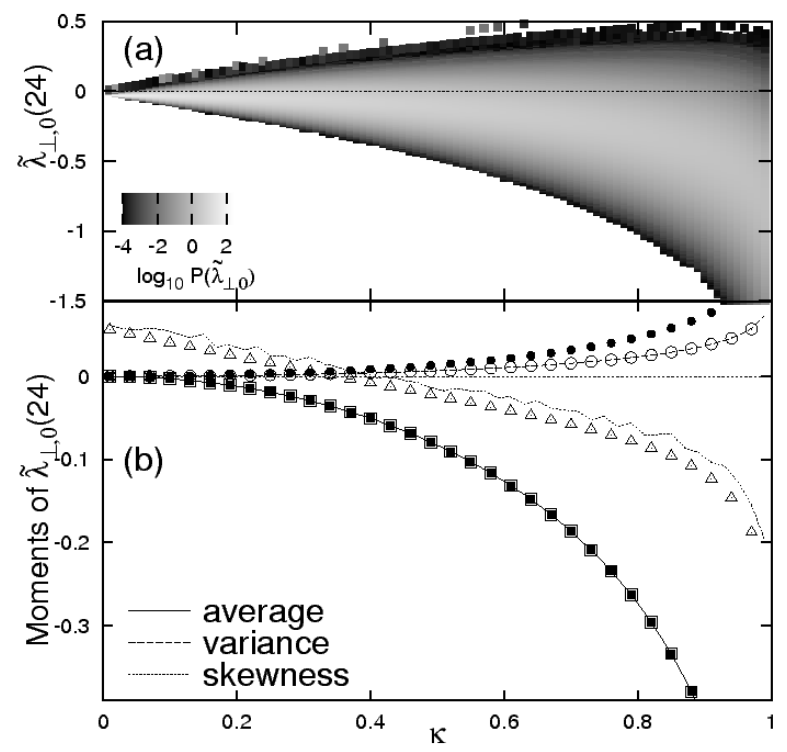

Figure 6 - (a) PDF (in gray-scale) and (b) some of its moments, for time-24 transversal Lyapunov exponents for typical orbits in the subspaces $y=0$ as a function of the parameter $\kappa$. The lines stand for typical chaotic orbits, whereas the symbols represent atypical unstable period- $p$ orbits (open symbols of $p=24$, filled symbols for $p=12$ ).

The dependence of the positive fraction of time- $n$ transverse Lyapunov exponents with $\kappa$ is depicted in Figure 7(a) for three different values of $n$. The results indicate that, for $0<\kappa<1$ we have a nonzero positive fraction of transversal exponents, on account of the existence of transversely unstable periodic orbits. Since the infinite-time Lyapunov exponent is already negative for this range of parameters there follows that the map always fulfill the conditions for riddling. This result holds for both attractors, so we have intermingled basins for any $\kappa$.

Our conclusions are reinforced by computing the so-called contrast measure, that quantifies the relative contribution of the unstable period- $p$ orbits to the natural measure of a chaotic attractor [28],

$$
C_{p}=\left|\mu_{p}^{u}-\mu_{p}^{s}\right|,
$$

where $\mu_{p}^{u}$ and $\mu_{p}^{s}$ are the contributions of period- $p$ orbits, embedded in the chaotic attractor, which are transversely unstable and stable, respectively. Riddling occurs when the former contribution is nonzero, hence the contrast measure is supposed to 
take on values between zero and unity for systems possessing riddled basins. The contrast measure is depicted in Figure 7(b) as a function of the parameter $\kappa$ for orbits with three different periods. The results confirm those obtained with help of finite-time Lyapunov exponents.

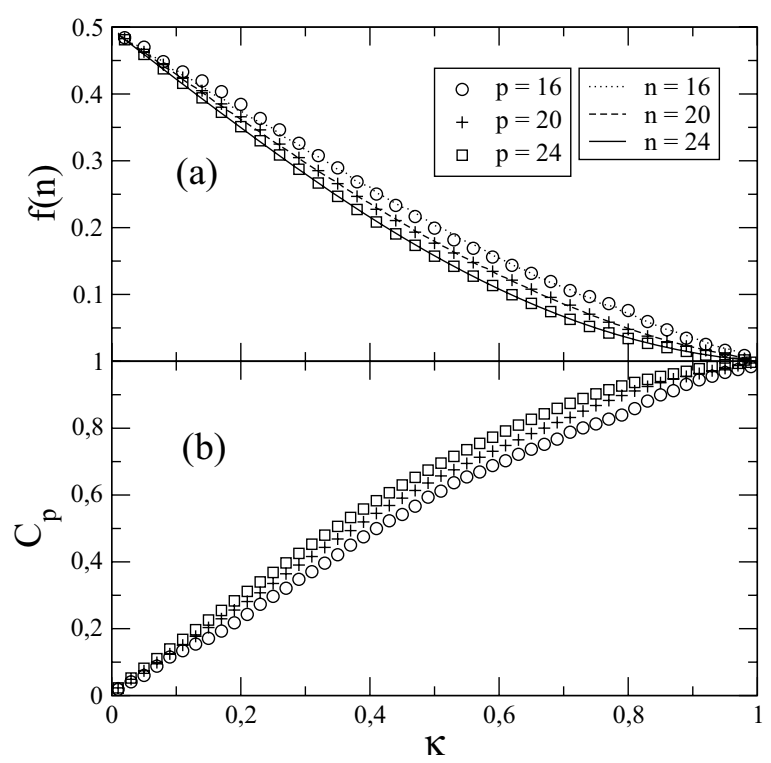

Figure 7 - Dependence with $\kappa$ of (a) the positive fraction of transversal Lyapunov exponents of attractor A for typical chaotic orbits ( $n=16,20,24)$ and unstable periodic orbits ( $p=16,20,24)$; (b) contrast measure for $p=16,20,24$.

\section{CONCLUSIONS}

Riddled basins, when occurring in complex systems, present many challenges for theoretical and experimental investigations. The extreme sensitivity to initial conditions make those systems highly susceptible to uncertainties of parameter and state determination. On the other hand, the rather stringent mathematical conditions necessary for the occurrence of riddled or intermingled basins (chiefly the mandatory existence of invariant manifolds for each coexisting attractor) limit the horizon of dynamical systems to be investigated. For example, coupled chaotic systems commonly present one such invariant manifold - the synchronization subspace. However, other invariant subspaces would require additional symmetry properties that not all complex systems are able to exhibit.

In spite of these difficulties, it turns out that riddling is quite common in complex systems. In this paper we have shown two representative examples of riddling in systems of physical and biological interest. Coupled Lorenz-like piecewise-linear maps can be viewed as a toy model that is expected to emulate dynamical features of complex systems describing climate change. We have shown that there are wide parameter values (in this case, the strength of coupling between the systems) for which the basin of the chaotic synchronized attractor is riddled. The advantages here are that we can actually prove that the basins are riddled, by verifying the mathematical requirements for that, which may be unfeasible in a higher-dimensional model. Moreover, since we were able to prove riddling for such a low-dimensional system, it is well likely that higher-dimensional models would also display riddled basins.

The second example studied involves a model for the competition between two species of flour beetles, in which the final states are the extinction of either species. The discrete-time model we used for this problem is simple enough that the mathematical requirements for riddling can be proved to exist for any intensity of the competition between species. Moreover, the basins of both coexisting attractors are mutually riddled, or intermingled. A practical consequence of riddling, in this case, is that the outcome of the species competition is indeterminate, as observed in experiments with populations of Tribolium sp.. The conventional explanation for this phenomenon has been stochastic, both of genetic and demographic origin, but we showed that intermingled basins offer a new perspective on this phenomenon, since they provide a deterministic origin for the competitive indeterminacy, what can be used to analyze the evolution of other animal populations.

\section{ACKNOWLEDGMENTS}

This work was partially supported by CNPq, CAPES, FAPESP, and Fundação Araucária (Brazilian government agencies).

\section{REFERENCES}

[1] SANJUAN MAF, AGUIRRE J \& VIANA RL. 2009. Rev. Mod. Phys., 81: 333 .

[2] ALEXANDER JC, YORKE JA, YOU Z \& KAN I. 1992. Int. J. Bifurcat. Chaos, 2: 795.

[3] LAI YC \& GREBOGI C. 1995. Phys. Rev. Lett., 52: R3313.

[4] McDONALD SW, GREBOGI C \& OTT E. 1985. Physica D, 17: 125.

[5] OTT E \& SOMMERER JC. 1993. Nature, 365: 138.

[6] OTT E, SOMMERER JC, ALEXANDER JC, KAN I \& YORKE JA. 1993. Phys. Rev. Lett., 71: 4134.

[7] OTT E, ALEXANDER JC, KAN I, SOMMERER JC \& YORKE JA. 1994. Physica D, 76: 384.

[8] ASHWIN P, BUESCU J \& STEWART I. 1994. Phys. Lett. A, 193: 126. 
[9] HEAGY JF, CARROLL TL \& PECORA LM. 1994. Phys. Rev. Lett., 73: 3528.

[10] WOLTERING M \& MARKUS M. 1999. Phys. Lett. A, 260: 453.

[11] CAZELLES B. 2001. Chaos, Solit. \& Fract., 12: 301.

[12] NAKAJIMA H \& UEDA Y. 1996. Physica D, 99: 35-44.

[13] WOLTERING M \& MARKUS M. 2000. Chem. Phys. Lett., 321: 473-478.

[14] YOUSEFI S, MAISTRENKO Y \& POPOVICH S. 2000. Discrete Dynamics in Nature and Society, 5: 161.

[15] HASLER M \& MAISTRENKO YL. 1997. IEEE Trans. Circ. Syst, I 44: 856.

[16] LORENZ E. 1963. J. Atmospheric Sciences, 20: 130.

[17] STEEB W-H, VAN WYK MA \& STOOP R. 1998. Int. J. Theor. Phys., 37: 2653.

[18] STOOP R \& STEEB W-H. 1997. Phys. Rev. E, 55: 7763.

[19] KIM C-M, RIM S, KYE W-H, RYU J-W \& PARK Y-J. 2003. Phys. Lett. A, 320: 39.
[20] FUJISAKA H \& YAMADA T. 1983. Prog. Theor. Phys., 69: 32.

[21] PECORA LM \& CARROLL TL. 1990. Phys. Rev. Lett., 64: 821.

[22] PARK T. 1954. Physiol. Zool., 27: 177.

[23] COSTANTINO RF \& DESCHARNAIS RA. 1991. Population dynamics and the Tribolium model: genetics and demography. (Springer Verlag, Berlin, 1991), Chap. 8

[24] COSTANTINO RF, CUSHING JM, DENNIS B \& DESHARNAIS RA. 1997. Nature, 375: 227. COSTANTINO RF, DESHARNAIS RA, CUSHING JM and DENNIS B. 1997. Science, 275: 389.

[25] HOFBAUER F, HOFBAUER J, RAITH P \& STEINBERGER T. 2004. J. Math. Biol., 49: 293.

[26] KAN I. 1994. Bull. Am. Math. Soc., 31: 68.

[27] PEREIRA RF, PINTO SES, VIANA RL, LOPES SR \& GREBOGI C. 2007. Chaos, 17: 023131.

[28] LAI Y-C. 1999. Phys. Rev. E, 59: R3807. 